\title{
Cladribine in the Treatment of Langerhans Cell Histiocytosis: A Summary of the Literature
}

\begin{abstract}
Langerhans cell Histiocytosis ( $\mathrm{LCH}$ ) is a rare disease for which treatment with 2chlorodeoxyadenosine (cladribine) has changed disease outcome. Cladribine has meanwhile been administered over two decades and is available for intravenous and subcutaneous administration. Cladribine has been administered in treatment of therapy naïve, relapsed and refractory LCH and induces high response rates, regardless of prior treatment. Literature describing the use of cladribine either alone or in combination with cytarabine is summarized herein.
\end{abstract}

Keywords: Cladribine; Langerhans cell histiocytosis; Summary
Marlies E.H.M. van Hoef*

Corresponding Author:

Marlies E.H.M. van Hoef

戸vanhoef@vanhoefconsulting.com

Citation: Van Hoef MEHM (2019) Cladribine in the Treatment of Langerhans Cell Histiocytosis: A Summary of the Literature. J Rare Disorders Diagnosis \& Therapy. Vol. 5 No. 1: 1.

Received: February 28, 2019; Accepted: March 14, 2019; Published: March 21, 2019

\section{Introduction}

$\mathrm{LCH}$ is a rare disease involving clonal proliferation of Langerhans cells, which are abnormal cells derived from the bone marrow, along with eosinophils, macrophages, lymphocytes and multinucleated giant cells. The incidence is 2 to 10 cases per one million children younger than 15 years, but it also occurs in adults [1]. Clinical manifestations range from isolated skin or bone lesions to multisystem disease. In LCH specific organs are considered: low risk (skin, bone, lymph nodes, pituitary gland), intermediate risk (lung, central nervous system) or high risk (liver, spleen, bone marrow) [2-4]. Treatment decisions are based on whether or not low, intermediate or high risk organs are involved and LCH presents as single site or multisystem disease [2-4]. In the guidelines for children up to eighteen years with $\mathrm{LCH}$, cladribine monotherapy is recommended in first line treatment for $\mathrm{LCH}$ of the lung and $\mathrm{LCH}$ brain lesions [3]. Cladribine monotherapy is furthermore recommended as second line treatment in LCH with low risk organ involvement and in combination with cytarabine when high risk organs are involved [3]. In the management recommendations for adult patients with LCH cladribine monotherapy is recommended in front line treatment of systemic therapy of single-system LCH with special site lesions, multifocal lesions and for multi-system LCH with or without involvement of high risk organs [4]. Cladribine is also recommended as treatment option when maintenance therapy is required, in refractory disease with or without cytarabine and in single or multisystem reactivation of $\mathrm{LCH}$ and furthermore in central nervous system involvement, regardless whether there are tumorous lesions or neurodegenerative disease [4]. Cladribine (2-chlorodeoxyadenosine) is a purine analog that was developed in the 1970s. It was first tested in humans in the early 1980s and became an established product for the treatment of Langerhans cell histiocytosis (LCH). Cladribine is a chemotherapeutic compound that can be administered intravenously or subcutaneously. It is a prodrug that is activated after uptake in cells [5]. Its functions as an antimetabolite that induces DNA strand breaks and is toxic in hematopoietic cells and leukemic and lymphatic malignancies, but has little or no effect in non-hematopoeitic tissues and solid tumors [5]. It is polyvalent and is toxic for dividing and quiescent cells [5]. Cladribine induces myelosuppression and immunosuppression. A summary of the efficacy of cladribine in LCH is described in this paper.

\section{Methods}

Literature review in pubmed was performed using the search terms cladribine and $\mathrm{LCH}$. Publications in which patients were treated with cladribine were identified and are included in this summary. Several publications that reported more patients reported case reports. There were also several case reports. As the disease manifests itselves heterogeneous and follow-up of response was variable, it was decided to develop a summary rather than a review, such that the reader can read through the various reports and get detailed information. This summary is composed of tables with articles; the text sections provides 
descriptions of the various articles listed in the tables. This is the first summary, cq review reported in the literature.

\section{Studies and Case Reports on Cladribine for LCH}

Table 1: Studies and case reports of children with LCH who were treated with cladribine.

Donadieu et al. [6] reported twenty seven children in whom $\mathrm{LCH}$ was diagnosed at the age between 0.08 and 3.34 years. At the time of treatment with cladribine and cytarabine patients had multiorgan involvement. Patients were refractory to vinblastine and steroids. Cladribine was administered at a dose of $9 \mathrm{mg} /$ $\mathrm{m}^{2}$ as two-hour infusion for five days. For children less than ten kilogram the dose was $0.3 \mathrm{mg} / \mathrm{kg} / \mathrm{d}$. Cytarabine was administered concurrently at a dose of $500 \mathrm{mg} / \mathrm{m}^{2}$ twice daily over two hours for five days. Up to five courses were administered until response occurred, whereafter seventeen patients received maintenance treatment consisting of two courses cladribine at $5 \mathrm{mg} / \mathrm{m}^{2} / \mathrm{d}$ for three days with vinblastine, prednisone, 6-mercaptopurine and methotrexate. The latter two drugs were continued orally for twelve more months. The overall response rate was $92 \%$. Twenty three patients $(85 \%)$ received the non-active disease status. Two patients died and in two patients the disease status was never considered controlled. Relapse occurred in six patients at a median of 0.9 years. Two patients relapsed in the skin for which vinblastine and 6-mercaptopurine was administered and four patients in other organs. One of these four patients received cladribine and died; the other three patients received cladribine and cytarabine and obtained a response after which two patients were transplanted. At five years follow-up overall survival was $85 \%$.

Mottl et al. [7] reported thirteen children with multisystem LCH. Median pretreatment duration was 15.7 months. Cladribine was used as salvage therapy. The first four patients received cladribine $0.1 \mathrm{mg} / \mathrm{kg} / \mathrm{d}$ continuously i.v. for seven days and nine patients 5 $\mathrm{mg} / \mathrm{m}^{2} / \mathrm{d}$ continuously i.v. for five days every three weeks during maximal six courses. Nine patients obtained complete remission after cladribine treatment alone and four patients received additional radiotherapy, maintenance with vinblastine and radiotherapy or maintenance with vinblastine, mercaptopurine and prednisone. At median follow-up of 5 years and 7 months all patients were alive and in complete remission.

Stine et al. [8] reported ten children with LCH with or without high risk organ involvement. Nine of them had been pretreated with one to four therapies. Treatment with cladribine was instated at a dose of $5 \mathrm{mg} / \mathrm{m}^{2} / \mathrm{d}$ for 3 days. If treatment was well tolerated the dose was increased to $6.5 \mathrm{mg} / \mathrm{m}^{2} / \mathrm{d}$ for 3 days for a maximum of six cycles in total. Treatment in nine patients was given on an outpatient basis. One patient withdrew from the study after three cycles and received six cycles vincristine and cytarabine and was free of disease at 16 months follow-up. One patient had progressive bone lesions and received thalidomide for one year and was disease free at 32 months follow-up. Nine patients received a minimum of four courses and were included in the evaluation. Eight patients obtained a complete response (88.9\%) and one a mixed response. The tenth patient is free of disease after thalidomide treatment. At 5 years follow-up seven patients $(70 \%)$ remained disease free and three patients relapsed or had reactivation of persistent distant disease.

Biswas et al. [9] reported seven children with relapsed or refractory $\mathrm{LCH}$, with multifocal bone lesions [3], multisystem low risk [2] and multisystem high risk [2] disease; skin, bone, lung, liver and lymphnodes were involved organs. Three patients had received one prior chemotherapy regimen and four were heavily pretreated. Patients received cladribine $0.15-0.3 \mathrm{mg} / \mathrm{kg} / \mathrm{d}$ over two hours for 5 days for median 6 (range 2-9) courses. Six patients were evaluable. Two (33\%) obtained partial remission and two (33\%) stable disease with clinical improvement. At median 19 months follow-up five of six evaluable patients were alive. Toxicity was mainly hematological but one patient was admitted with diarrhea and sepsis and died.

Rodriguez et al. [10] reported six children with relapsed multifocal or multisystem $\mathrm{LCH}$. Patients received cladribine $5-7 \mathrm{mg} / \mathrm{m}^{2} / \mathrm{d}$ by two hour intravenous infusion for five days every 3 to 4 weeks for maximal six courses. All patients received a complete response. At a median follow-up of 15 months five patients (83.3\%) remained in remission. One patient with multisystem disease and relapse obtained a second response with cladribine and was on the treatment at the time of the report.

Stine et al. [11] reported three children with $\mathrm{LCH}$ with multiple organ involvement. One patient $(2.9 \mathrm{y})$ received her first course of cladribine at a dose of $8 \mathrm{mg} / \mathrm{m}^{2} / \mathrm{d}$ by continuous infusion for five days. Subsequent courses were reduced to $5 \mathrm{mg} / \mathrm{m}^{2} / \mathrm{d}$ by continuous infusion for 3 days every 3 to 4 weeks for three courses. The second patient received cladribine $6.5 \mathrm{mg} / \mathrm{m}^{2} / \mathrm{d}$ continuous i.v. for 3 days for six courses at 4 weeks interval. The third patient received cladribine at not reported dose for five courses at 4 weeks interval. All three patients obtained complete remission and were in remission at respectively 18,12 and 14 months follow-up.

Baumann et al. [12] reported a twenty year old patient with multisystem $\mathrm{LCH}$ who had been treated with vinca-alkaloid therapy and sorafenib; the latter induced a partial remission. Upon relapse the patient was treated with cladribine $10 \mathrm{mg} / \mathrm{d}$ subcutaneously for five days every 3 weeks for four cycles. After four months the patient received two additional cycles because of visual field impairment and obtained complete remission, which lasted for $18+$ months. The plan was to retreat with cladribine if relapse would occur, as the patient had a long disease free period.

Ottaviano et al. [13] reported a three year old child with diabetes insipidus, who had presented with cutaneous eruptions at 2 months of age. At 14 months of age he was diagnosed with LCH. He was treated with vinblastine and prednisone, 6-mercaptopurine and 6-thioguanine with prednisone for skin relapses and topical tacrolimus. When he developed diabetes insipidus cladribine 0.3 $\mathrm{mg} / \mathrm{kg} / \mathrm{d}$ for 3 days was initiated. Five cycles were administered. At 18 months follow-up the patient was free of diabetes insipidus and other manifestations of LCH.

Suzuki et al. [14] reported a one year old boy who presented with an occipital tumor diagnosed as $\mathrm{LCH}$. He was treated with cytarabine, vincristine and prednisolone which induced a 
Table 1: Studies and case reports of children with LCH who were treated with cladribine.

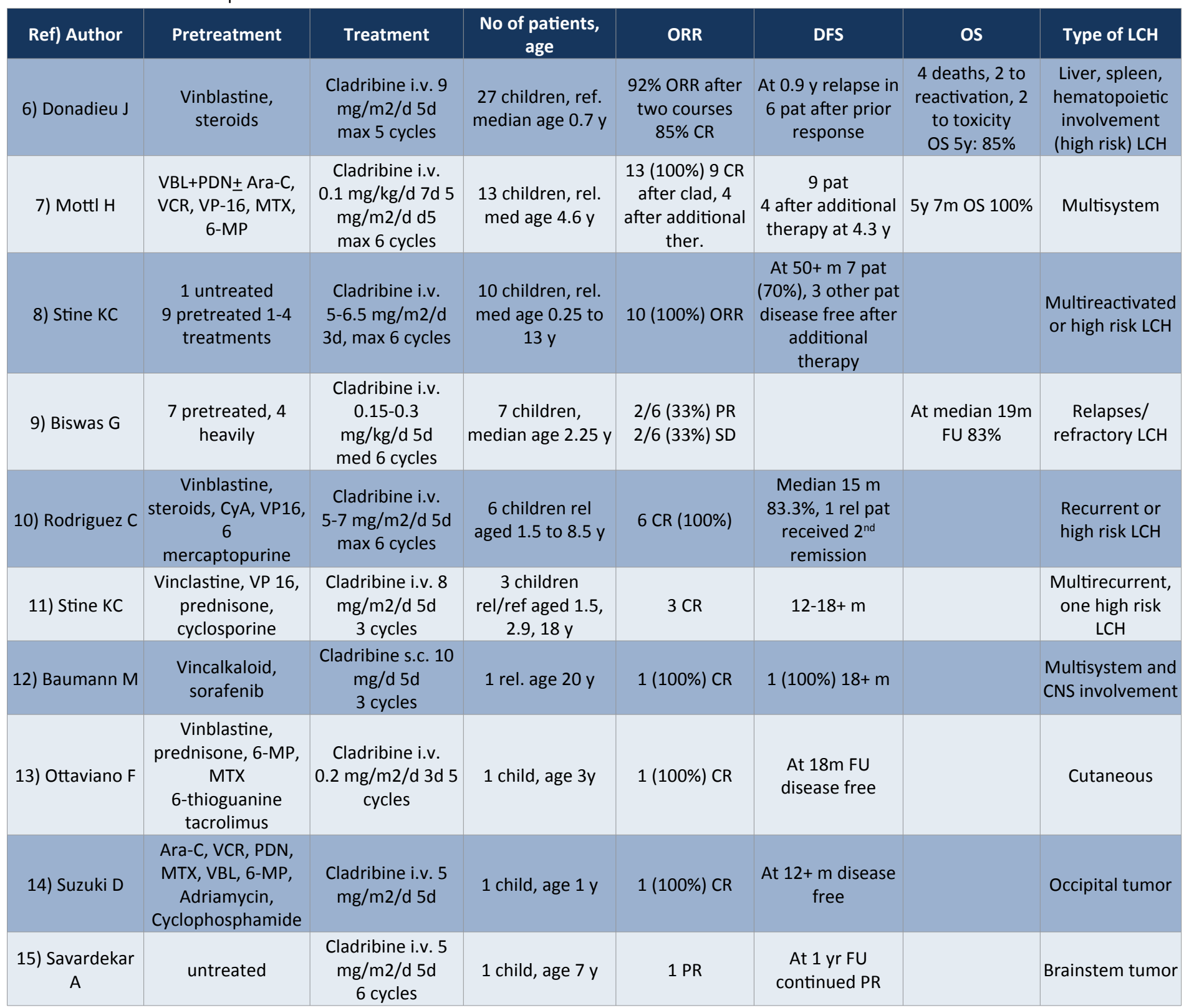

$\mathrm{ORR}=$ overall response rate, $\mathrm{DFS}=$ disease free survival, $\mathrm{OS}=$ overall survival, $C R=$ complete response, $P R=$ partial response, $S D=$ stable disease, $F U=$ followup, $V B L=$ vinblastine, $P D N=$ prednisone, Ara- $C=$ cytarabine, $V C R=$ vincristine, $V P=16=$ etoposide, $M T X=$ methotrexate, 6-MP=6-mercaptopurine, $\mathrm{CyA}=$ cyclosporine, i.v.=intravenous, $s . c=$ subcutaneous, rel=relapsed, ref=refractory, $m=$ month, $y=y e a r, C N S=$ central nervous system

complete response and maintenance with vincristine, cytarabine, prednisolone, methotrexate, vinblastine and 6-mercaptopurine. The tumor relapsed in the left orbital cavity and he subsequently received vincristine, adriamycine, cyclophosphamide and cyclosporine. A relapse in both locations occurred for which he was treated with cladribine $5 \mathrm{mg} / \mathrm{m}^{2} / \mathrm{d}$ for 5 days every three weeks. Six months later he developed myelodysplastic syndrome (MDS), which spontaneously regressed. At more than one year follow-up he was free of MDS and LCH.

Savardekar et al. [15] reported a seven year old boy who presented with facial paresis. Physical examination revealed abducent, facial and vestibulocochlear nerve involvement. The bilateral pyramidal tracts were involved and induced hypotonia and exaggerated deep tendon reflexes in all four limbs. The patient furthermore had gait ataxia. The diagnosis of LHC was made. Cladribine was started at a dose of $5 \mathrm{mg} / \mathrm{m}^{2} / \mathrm{d}$ over two hours intravenously for 5 days during six courses. A partial remission was obtained. At one year follow-up the partial remission continued, though with neurological sequela.

Table 2: Studies and case reports of children with LCH who were treated with cladribine and cytarabine.

Rigaud et al. [16] reported 1478 patients with LHC, aged less than 18 years, from the French registry, which was established in 1983. Registry assessment revealed that patients had less severe disease by time. In 1998 a change in treatment practice occurred: 483 Patients were diagnosed before and 995 patients after 1998. Vinblastine and steroids were given and salvage therapy was not 
standardized before 1998; in 1998 treatment practice changed: therapy duration increased from 6 to 12 months, repeated induction therapy was administered in cases showing poor response to the first induction with vinblastine and steroids, and refractory disease in patient with high risk organ involvement was treated with cladribine and cytarabine. Also maintenance treatment was prolonged from 6 to 12 months. Two induction treatments with vinblastine and steroids were given to $9.2 \%$ of patients before 1998 and to $22 \%$ of patients after 1998 . In total 42 patients $(5 \%)$ were treated with cladribine and cytararabine combination therapy. At five years the relapse rate was $47 \%$ in patients treated before 1998 and 33\% in patients treated after 1998. At 5 years the relapse rate reached a plateau, although late relapses were occasionally observed. The 5 years and 10 years overall survival rates were respectively $92.3 \%$ and $91.9 \%$ before 1998 and $98.7 \%$ and $98.7 \%$ after 1998 . Patients with involvement of risk organs had a 10 year survival of $49.8 \%$ before 1998 and of $91.7 \%$ after 1998. Following the introduction of cladribine and cytarabine treatment for refractory patients with involvement of risk organs, survival increased from $27 \%$ to $83 \%$. Single system or multisystem $\mathrm{HCL}$ patients without involvement of risk organs showed survival rates of $100 \%$ over time.
$\mathrm{Hu}$ et al. [17] reported thirteen children with refractory multisystem LCH. Two patients with liver cirrhose stopped treatment after one cycle of therapy and one patient died of sepsis. The treatment was recommended as salvage therapy after failure of first line therapy, and as first line therapy for multisystem LCH with risk organ involvement.

Bernard et al. [18] reported ten childeren of young age with refractory LCH. At diagnosis all patients had skin, liver and spleen involvement and hematopoietic dysfunction. Seven patients had hemophagocytic syndrome, associated in six with viral infections, three had bone lesions and two presented with gut involvement. Patients received cytarabine $1000 \mathrm{mg} / \mathrm{m}^{2} / \mathrm{d}$ by two-hour infusions and cladribine $9 \mathrm{mg} / \mathrm{m}^{2} / \mathrm{d}$ as continuous intravenous infusion for five days. Cladribine was administered at a dose of $0.3 \mathrm{mg} / \mathrm{kg} / \mathrm{d}$ if weight was below $10 \mathrm{~kg}$. Two to four courses were administered at 4 weeks interval and additional therapy was given on an individual basis and consisted of cladribine monotherapy, retinoid acid, or cladribine with vinblastine and steroids. Seven patients were evaluable for response, six obtained an early partial response and one responded later. Two patients died from sepsis and one after an additional allogeneic transplant. At three years

Table 2: Studies and case reports of children with LCH who were treated with cladribine and cytarabine.

\begin{tabular}{|c|c|c|c|c|c|c|c|}
\hline Ref) Author & Pretreatment & Treatment & $\begin{array}{c}\text { No of patients, } \\
\text { age }\end{array}$ & ORR & DFS & OS & Type pf LCH \\
\hline 16) Rigaud C & $\begin{array}{l}\text { Vinblastine, } \\
\text { steroids }\end{array}$ & $\begin{array}{c}\text { Cladribine i.v. }+ \\
\text { Cytarabine }\end{array}$ & $\begin{array}{l}1478 \mathrm{rel} / \mathrm{ref} \\
\text { aged }<18 \mathrm{y} \\
42 \text { patients } \\
\text { treated with } \\
\text { combination }\end{array}$ & & $\begin{array}{c}\text { At } 5 \text { y before } \\
199853 \%, \\
\text { At } 5 \text { y after } 1998 \\
67 \% .\end{array}$ & $\begin{array}{l}\text { OS } 5 \text { y } 96.6 \% \\
\text { improving from } \\
92.3 \% \text { to } 98.7 \% \\
\text { after } 1998 \text { in all }\end{array}$ & $\begin{array}{c}\text { OS } 5 \text { y improving } \\
\text { from } 49.8 \% \text { to } \\
91.7 \% \text { in high risk } \\
\text { patients after } \\
1998\end{array}$ \\
\hline 17) $\mathrm{Hu} \mathrm{T}$ & Pretreated & $\begin{array}{l}\text { Cladribine i.v. + } \\
\text { cytarabine }\end{array}$ & $\begin{array}{l}13 \text { children, } \\
\text { ref. }\end{array}$ & 10 (77\%) ORR & & & $\begin{array}{l}\text { Refractory high } \\
\text { risk or recurrent }\end{array}$ \\
\hline 18) Bernard F & $\begin{array}{c}\text { Vincristine, } \\
\text { steroids, VP16, } \\
\text { MTX }\end{array}$ & $\begin{array}{c}\text { Cladribine i.v. } \\
9 \mathrm{mg} / \mathrm{m} 2 / \mathrm{d} 5 \mathrm{~d}+ \\
\text { cytarabine } 1 \mathrm{~g} / \mathrm{m} 2 / \mathrm{d} \\
2-4 \text { cycles }\end{array}$ & $\begin{array}{c}10 \text { children, } \\
\text { ref. } \\
\text { age } 0.4 \text { to } 1.5 \mathrm{y}\end{array}$ & $\begin{array}{l}6 / 7(86 \%) \text { ORR } \\
\text { with additional } \\
\text { therapy given to } \\
\text { some patients }\end{array}$ & & 3 у OS $70 \%$ & $\begin{array}{l}\text { Refactory and } \\
\text { hematopoitic } \\
\text { dysfunction }\end{array}$ \\
\hline 19) Rosso DA & $\begin{array}{c}\text { Vinblastine, } \\
\text { sterioids, } \\
\text { methotrexate }\end{array}$ & $\begin{array}{c}\text { Cladribine i.v. } \\
5 \mathrm{mg} / \mathrm{m} 2 / \mathrm{d} 5 \mathrm{~d}+ \\
\text { cytarabine } 1 \mathrm{~g} / \mathrm{m} 2 / \mathrm{d} \\
5 \mathrm{~d} \\
4 \text { cycles }\end{array}$ & $\begin{array}{l}9 \text { children, } \\
\text { median age } \\
0.75 \mathrm{y}\end{array}$ & $\begin{array}{c}6(67 \%) \text { CR } \\
7(77.8 \%) \text { ORR }\end{array}$ & $\begin{array}{l}\text { At median } 6.5 \text { y } \\
\text { FU DFS } 77.8 \%\end{array}$ & $\begin{array}{l}\text { At } 3 \text { y probability } \\
\text { OS } 0.73 \pm 0.16\end{array}$ & $\begin{array}{c}5 \text { kids } \\
\text { continuation ther, } \\
3 \text { other ther }\end{array}$ \\
\hline $\begin{array}{l}\text { 20) Apollonsky } \\
\mathrm{N}\end{array}$ & $\begin{array}{l}\text { Vinblastine, } \\
\text { steroids, } \\
2 \text { children } \\
\text { cladribine } \\
\text { monotherapy }\end{array}$ & $\begin{array}{l}\text { Cladribine i.v. } \\
8.9 \mathrm{mg} / \mathrm{m} 2 / \mathrm{d} 5 \mathrm{~d} \\
+ \text { cytarabine } 1 \mathrm{~g} / \\
\mathrm{m} 2 / \mathrm{d} 5 \mathrm{~d} \\
3-5 \text { cycles }\end{array}$ & $\begin{array}{c}5 \text { children, } 4 \\
\text { younger than } \\
2 y\end{array}$ & $5(100 \%)$ ORR & $\begin{array}{l}\text { At } 2.5 \text { to } 6 \text { y FU } \\
\text { DFS } 100 \%\end{array}$ & & $\begin{array}{l}\text { Recurrent (3) and } \\
\text { high risk (2) } \mathrm{LCH}\end{array}$ \\
\hline 21) Choi SW & Untreated & $\begin{array}{l}\text { Cladribine i.v. + } \\
\text { VBL + prednison } \\
\text { Cladribine i.v. + } \\
\text { HD cytarabine }\end{array}$ & $\begin{array}{l}1 \text { child, age } \\
8 \text { m with GI } \\
\text { involvement }\end{array}$ & $\begin{array}{c}1 \mathrm{PR} \\
1 \text { durable CR }\end{array}$ & $27+m$ & & $\begin{array}{c}\text { Skin, gastro- } \\
\text { intestinal tract, } \\
\text { multisystem high } \\
\text { risk }\end{array}$ \\
\hline \multirow[t]{2}{*}{ 22) Yao $X$} & Pretreated & $\begin{array}{c}\text { Cladribine i.v. } 10 \\
\mathrm{mg} / \mathrm{m} 2 / \mathrm{d} 4 \mathrm{~d} \\
+ \text { cytarabine c3- }\end{array}$ & 1 child, age 6 y & $1(100 \%) C R$ & $\begin{array}{c}\text { At } 8 \mathrm{~m} \text { disease } \\
\text { free }\end{array}$ & & $\begin{array}{c}\text { Cytarabine in } 3 \\
\text { of } 5 \text { cycles }\end{array}$ \\
\hline & & $\begin{array}{c}5 \\
5 \text { cycles }\end{array}$ & & & & & \\
\hline
\end{tabular}

ORR=overall response rate, $D F S=$ disease free survival, OS=overall survival, $C R=$ complete response, $V P-16=e$ oposide, $M T X=m e t h o t r e x a t e$, $\mathrm{VBL}=$ inblastine, $\mathrm{HD}=$ high dose, i.v.=intravenous, rel=relapsed, ref=refractory, $\mathrm{m}=\mathrm{month}, \mathrm{y}=\mathrm{year}, \mathrm{Gl}=\mathrm{gastrointestinal}$ 
follow-up overall survival was $70 \%$, no patient had active disease and all had almost normal blood parameters.

Rosso et al. [19] reported nine children with median age of 0.75 years with multisystem high risk $\mathrm{LCH}$. All patients had hepatosplenomegaly, hematological involvement and transfusion requirements. When they progressed after treatment consisting of vinblastine and prednisone with or without methotrexate, they received five or six courses of cladribine $5 \mathrm{mg} / \mathrm{m}^{2} / \mathrm{d}$ for 5 days and cytarabine $100 \mathrm{mg} / \mathrm{m}^{2} / \mathrm{d}$ for 4 days. Six patients obtained complete response and one patient improvement of active disease; five patient received continuation therapy and three who showed reactivation received other agents. At median follow-up of 6.5 years seven patients were disease free. Two patients died due to progressive disease. The probability of survival at three years was 0.73 .

Apollonsky et al. [20] reported five young children with multisystem LCH. Two patients had high risk disease with lung, liver and spleen involvement. All patient had received mean 43 weeks of standard chemotherapy with vinblastine and steroids. They soon relapsed or progressed. Two patients received radiation and two cladribine monotherapy. In view of subsequent relapse all patients received cladribine $8.9 \mathrm{mg} / \mathrm{m}^{2} / \mathrm{d}$ $(0.3 \mathrm{mg} / \mathrm{kg} / \mathrm{d}$ for children less than $10 \mathrm{~kg})$ continuously i.v. for 5 days and cytarabine $1 \mathrm{~g} / \mathrm{m}^{2} / \mathrm{d}$ over two hours for 5 days during median four cycles. All patients obtained complete or partial remission. During 2.5 to 6 years follow-up all patients remained free of disease.

Choi et al. [21] reported a child of 8 months with LCH with enterocolitis, massive splenomegaly, rash, pancytopenia, hypoproteinemia and hypoalbuminemia. The patient received two cycles of cladribine which led to transient improvement of diarrhea and rash. During the subsequent two cycles vinblastine and corticostoids were added after which gastrointestinal improvement was observed. The patient then received three chemotherapy cycles. He was subsequently readmitted with fever and neutropenia and recurrence of symptoms. Administration of two cycles of cladribine with high dose cytarabine resulted in complete resolution of all signs and symptoms of disease activity. The patient was free of disease 35 months from initial diagnosis and 27 months after the last chemotherapy.

Yao et al. [22] reported a six year old patient who had been diagnosed with $\mathrm{LCH}$ at three months of age. He had skin, skeleton, lung and liver involvement and relapsed three times on treatment before he was treated with cladribine. The dose was $10 \mathrm{mg} / \mathrm{m}^{2} / \mathrm{d}$ for 4 days. Five courses were administered, and during the third to fifth course cytarabine was added. The patient obtained complete remission and was disease free at 8 months follow-up.

Table 3: Studies and case reports of adults with LCH who were treated with cladribine.

Cantu et al. [23] reported fifty eight adults with a mean age of 32 years, who were treated with three chemotherapy regimen for bone lesions either solitary or as component of multisystem disease. Twenty two patient received cladribine $5 \mathrm{mg} / \mathrm{m}^{2} / \mathrm{d}$ intravenously for 5 days during six courses. Nine patients $(41 \%)$ responded to treatment in first year. Compared to vinblastine and prednisone (ORR 16\%) and low dose cytarabine (ORR 79\%), the response to cladribine was $41 \%$.

Saven et al. [24] reported thirteen adults with $\mathrm{LCH}$ with involvement of skin, bone, lung, soft tissue and/or lymph nodes. Twelve of them had been pretreated with steroids, radiotherapy and/or chemotherapy. The median disease duration prior to treatment with cladribine was ninety nine months. The first two patients received cladribine $0.1 \mathrm{mg} / \mathrm{kg} / \mathrm{d}$ continuously i.v. for 7 days and eleven patients received cladribine $0.14 \mathrm{mg} / \mathrm{kg} / \mathrm{d}$ by 2-hour infusion for 5 days. Courses were repeated every 4 weeks for a maximum of six courses. Twelve patients were evaluable for response; one patient was not seen again after the first course. After a median of three courses seven patients (58\%) obtained a complete response and two patients (17\%) a partial response for and overall response rate of $75 \%$. Three patients had stable disease. Median follow-up for all patients was 33 months, for patients in complete remission $33+$ months and for patients in partial remission $8+$ and $36+$ months. At a median follow-up of 42 months twelve patients $(92.3 \%)$ were alive.

Adam et al. [25] also reported ten adults who received treatment with cladribine. Eight had multiorgan involvement and two had multifocal skeletal lesions with aggressive course. Patients were either treatment naive or pretreated. Cladribine was administered at $5 \mathrm{mg} / \mathrm{m}^{2} / \mathrm{d}$ subcutaneously for 5 days every 4 weeks. In two patients there was insufficient result after three cycles and cladribine was combined with cyclophosphamide and dexamethasone. Maximal six cycles were administered. After a median of five cycles nine patients $(90 \%)$ obtained complete remission and were disease free at a median of 26 months. The patient who progressed after 60 days received cyclophosphamide, doxorubicin, vincristine, etoposide and prednisone (CHOEP) and high dose carmustine, etoposide, cytarabine and melphalan (BEAM) with autologous transplantation.

Adam et al. [26] retrospectively studied seven treatment naive adult males, six with multisystem and one with multifocal bone $\mathrm{LCH}$, who were treated with cladribine, administered as 2-hour infusion or as subcutaneous injection, $5 \mathrm{mg} / \mathrm{m}^{2} /$ day for 5 days at 4 week intervals for four courses. Two patients with aggressive disease underwent stem cell mobilization with cyclophosphamide, etoposide and G-CSF for potential autologous transplantation prior to start of cladribine. One of these patients responded to cladribine and did not require further therapy, the other was refractory and required autologous and allogeneic transplantation. In two patients with insufficient response after two to three cycles, cladribine treatment was enhanced with cyclophosphamide and steroids. Three patients received concomitant radiotherapy and one patient PUVA. Durable complete remissions were obtained in six patients $(86 \%)$ and the patients were disease free at median follow- up of 37 months. One patient relapsed 2 months after cladribine treatment.

Pardanani et al. [27] described five adult patients, who had disseminated $\mathrm{LCH}$, one with central nervous system involvement. One patient was treatment naïve and the other four had been pretreated. Cladribine was administered at a dose 0.09-0.1 mg/ $\mathrm{kg} / \mathrm{d}$ intravenously for 5 to 7 days during median four courses. 
Table 3: Studies and case reports of adults with LCH who were treated with cladribine.

\begin{tabular}{|c|c|c|c|c|c|c|c|}
\hline Ref) Author & Pretreatment & Treatment & $\begin{array}{c}\text { No of patients, } \\
\text { age }\end{array}$ & ORR & DFS & OS & Type of LCH \\
\hline 23) Cantu MA & untreated & $\begin{array}{c}\text { Cladribine i.v. } 5 \mathrm{mg} / \\
\mathrm{m} 2 / \mathrm{d} 5 \mathrm{~d} \\
6 \text { cycles }\end{array}$ & 22 adults & $9(41 \%)$ in 1 year & & & $\begin{array}{c}\text { Bone lesion or } \\
\text { bone and } \\
\text { multisystem }\end{array}$ \\
\hline 24) Saven A & $\begin{array}{l}\text { One untreated, } \\
\text { one predisone, } \\
\text { one RT, } \\
\text { six ChT + RT, } \\
\text { four surgery, ChT } \\
+\mathrm{RT}\end{array}$ & $\begin{array}{c}\text { Cladribine i.v. } \\
0.1 \mathrm{mg} / \mathrm{kg} / \mathrm{d} 7 \mathrm{~d} \\
0.14 \mathrm{mg} / \mathrm{kg} / \mathrm{d} 5 \mathrm{~d} \max \\
6 \text { cycles }\end{array}$ & $\begin{array}{c}13 \text { adults, } \\
\text { median age } 42 \text { y }\end{array}$ & $\begin{array}{c}12 \text { evaluable; } \\
\text { After med } 3 \text { courses } \\
7 \text { CR }(58 \%) \\
2 \text { PR }(17 \%) \\
\text { ORR } 75 \% \text { at } \\
\text { median } 33 \mathrm{~m}\end{array}$ & & $\begin{array}{l}\text { At median } \\
42 \mathrm{~m} 91.3 \% \text {. }\end{array}$ & $\begin{array}{l}\text { Multisystem, } \\
4 \text { also lung } \\
\text { involvement }\end{array}$ \\
\hline 25) Adam Z & $\begin{array}{l}\text { Partially no prior } \\
\text { treatment }\end{array}$ & $\begin{array}{c}\text { Cladribine s.c. } 5 \mathrm{mg} / \\
\mathrm{m} 2 / \mathrm{d} 5 \mathrm{~d} \\
\max 6 \text { cycles }\end{array}$ & $\begin{array}{c}10 \text { adults, } \\
\text { median age } 31.5 \\
y\end{array}$ & 9 CR (90\%) & $\begin{array}{c}9(90 \%) \text { at } \\
\text { median } 26 \mathrm{~m}\end{array}$ & & $\begin{array}{c}\text { Multifocal or } \\
\text { multisystem } \\
\text { LCH }\end{array}$ \\
\hline 26) Adam Z & Untreated & $\begin{array}{l}\text { Cladribine i.v. or s.c., } \\
5 \mathrm{mg} / \mathrm{m} 2 / \mathrm{d} \\
5 \mathrm{~d}, 4 \mathrm{cycles} \text { alone or } \\
\text { in combi with cyclo } \\
+ \text { dex }\end{array}$ & $\begin{array}{c}7 \text { adults, median } \\
\text { age } 34 \text { y }\end{array}$ & $\begin{array}{l}6(86 \%) C R \\
1(14 \%) P R\end{array}$ & $\begin{array}{c}6(86 \%) \text { at } \\
\text { median } 37 \mathrm{~m}\end{array}$ & & $\begin{array}{l}\text { Multifocal } \\
\text { (1) and } \\
\text { multisystem } \\
\text { (6) LCH }\end{array}$ \\
\hline 27) Pardanani $A$ & $\begin{array}{l}1 \text { untreated } \\
4 \text { pretreated }\end{array}$ & $\begin{array}{c}\text { Cladribine i.v. 0,09- } \\
0.1 \mathrm{mg} / \mathrm{kg} / \mathrm{d} \\
5-7 \mathrm{~d}, 4 \text { cycles }\end{array}$ & $\begin{array}{c}5 \text { adults, age } 19 \\
\text { to } 81 \mathrm{y}\end{array}$ & $\begin{array}{l}3(60 \%) \text { CR } \\
\text { ORR 100\% }\end{array}$ & $\begin{array}{l}\text { At median } 33 \mathrm{~m} \\
\text { DFS/PFS } 80 \% .\end{array}$ & & $\begin{array}{l}\text { Disseminated } \\
\text { LCH, } 1 \text { CNS } \\
\text { involvement }\end{array}$ \\
\hline 28) Schini M & Pretreated & $\begin{array}{l}\text { Cladribine i.v. } \\
0.14 \mathrm{mg} / \mathrm{kg} / \mathrm{d} 5 \mathrm{~d} \\
\text { at least } 4 \text { cycles }\end{array}$ & $\begin{array}{c}5 \mathrm{rel} / \mathrm{ref} \text { adults, } \\
\text { age }>18 \mathrm{y}\end{array}$ & $\begin{array}{c}\text { ORR } 80 \% \\
1 \text { sustained SD }\end{array}$ & $\begin{array}{l}\text { At median } 56 \mathrm{~m} \\
\text { PFS } 100 \%\end{array}$ & & $\begin{array}{l}\text { Disseminated } \\
\text { LCH, } 1 \\
\text { cerebellar } \\
\text { involvement }\end{array}$ \\
\hline 29) Saven A & $\begin{array}{cc}1 & \text { untreated } \\
2 & \text { pretreated }\end{array}$ & $\begin{array}{l}\text { Cladribine i.v. } \\
0.7 \mathrm{mg} / \mathrm{kg} / \text { course } \\
2-5 \text { cycles }\end{array}$ & $\begin{array}{l}3 \text { adults, aged } \\
33,51 \text { and } 57\end{array}$ & $3(100 \%)$ CR & $\begin{array}{l}\text { At } 5,15 \text { and } 23 \\
\text { months DSF } \\
100 \%\end{array}$ & & $\begin{array}{l}2 \text { low risk, } 1 \\
\text { high risk patient }\end{array}$ \\
\hline 30) Vanicek J & 2 untreated & $\begin{array}{l}\text { Cladribine s.c. } \\
5 \mathrm{mg} / \mathrm{m} 2 / \mathrm{d} 5 \mathrm{~d} \\
4-6 \text { cycles }\end{array}$ & $\begin{array}{c}2 \text { adults, aged } \\
33 \\
y\end{array}$ & $2(100 \%) C R$ & $\begin{array}{c}\text { At } 44 \text { and } 8 \\
\text { m FU } \\
\text { DFS } 100 \%\end{array}$ & & $\begin{array}{l}\text { Diabetes } \\
\text { insipidus }\end{array}$ \\
\hline 31) Lajolo C & $\begin{array}{c}\text { Etoposide } \\
\text { Radiotherapy }\end{array}$ & $\begin{array}{c}\text { Cladribine } \\
0.14 \mathrm{mg} / \mathrm{kg} / \mathrm{d} 5 \mathrm{~d} \\
4 \text { cycles }\end{array}$ & $\begin{array}{c}1 \text { adults, aged } \\
77 y\end{array}$ & $1 \mathrm{CR}$ & & & $\begin{array}{l}\text { Palatal, vulvar, } \\
\text { parotis lesion }\end{array}$ \\
\hline $\begin{array}{l}\text { 32) Dimopoulos } \\
\text { MA }\end{array}$ & VP16 + steroids & $\begin{array}{l}\text { Cladribine i.v. } \\
0.1 \mathrm{mg} / \mathrm{kg} / \mathrm{d} 7 \mathrm{~d} \\
4 \text { cycles }\end{array}$ & $\begin{array}{l}1 \text { adult refr } \\
\text { aged } 34 \mathrm{y}\end{array}$ & $1 \mathrm{CR}$ & $12+m$ & & $\begin{array}{l}\text { Bones, bone } \\
\text { marrow, colon }\end{array}$ \\
\hline 33) Dallafior S & $\begin{array}{l}\text { Mechorethamin } \\
\text { Diprolen }\end{array}$ & $\begin{array}{l}\text { Cladribine i.v. } \\
0.1 \mathrm{mg} / \mathrm{kg} / \mathrm{d} \text { 7d } 1 \\
\text { cycle }\end{array}$ & $\begin{array}{c}1 \text { adult, aged } \\
65 \mathrm{y}\end{array}$ & $\begin{array}{l}1 \text { response with } \\
\text { recurrence after } 2 \\
\text { months }\end{array}$ & & & $\begin{array}{c}\text { Cutaneous LCH, } \\
\text { further treated } \\
\text { with } \\
\text { Thalidomide }\end{array}$ \\
\hline 34) Robak T & $\begin{array}{l}\text { Vincristine } \\
\text { Prednisone }\end{array}$ & $\begin{array}{c}\text { Cladribine i.v. } \\
0.12 \mathrm{mg} / \mathrm{kg} / \mathrm{d} 3 \mathrm{~d}+ \\
\text { Cyclophosphamide } \\
650 \mathrm{mg} / \mathrm{m} 2 \mathrm{~d} 1 \\
5 \mathrm{cycles}\end{array}$ & $\begin{array}{c}1 \text { adult, aged } \\
57 y\end{array}$ & $1 \mathrm{CR}$ & $\begin{array}{c}\text { At } 6 \mathrm{~m} \text { FU DFS } \\
100 \%\end{array}$ & & SLE and LCH \\
\hline 35) Buchler T & $\begin{array}{l}\text { Cytostatics HD } \\
\text { steroids } \\
\text { Radiotherapy }\end{array}$ & $\begin{array}{c}\text { Cladribine i.v. } 5 \mathrm{mg} / \\
\mathrm{m} 2 / \mathrm{d} 5 \mathrm{~d} \\
6 \text { cycles }\end{array}$ & $\begin{array}{c}1 \text { adult, aged } \\
25 y\end{array}$ & $1 \mathrm{ORR}$ & & & Brain stem \\
\hline 36) Black A & untreated & $\begin{array}{c}\text { Cladribine i.v. } 7 d \\
1 \text { cycles }\end{array}$ & $\begin{array}{c}1 \text { adult, aged } \\
79 \text { years }\end{array}$ & $1 \mathrm{CR}$ & & & Cutaneous LCH \\
\hline 37) Gabalec F & untreated & $\begin{array}{c}\text { Cladribine } \\
0.14 \mathrm{mg} / \mathrm{kg} / \mathrm{d} 5 \mathrm{~d} \\
5 \text { cycles }\end{array}$ & $\begin{array}{c}1 \text { adult, aged } \\
42 y\end{array}$ & $\begin{array}{l}1 \mathrm{CR} \text {, with new } \\
\text { jejunum lesion }\end{array}$ & & & $\begin{array}{c}\text { Systemic LCH } \\
\text { with suprasellar } \\
\text { lesion }\end{array}$ \\
\hline
\end{tabular}

ORR=overall survival, DFS=disease free survival, $P F S=$ progression free survival, $O S=$ overall survival, $C R=$ complete response, $P R=$ partia response, $\mathrm{SD}=$ stable disease, $\mathrm{FU}=$ follow-up, $\mathrm{RT}=$ radiotherapy, $\mathrm{ChT}=$ chemotherapy, i.v.=intravenous, s.c.-subcutaneous, combi=combination,

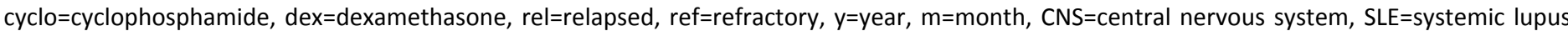
erythomatodes 
The patient with nervous system involvement obtained complete remission of symptoms and disease after five courses. Patients with skin lesions and lymphadenopathy, and skin, mucosal and lung involvement respectively obtained also complete remission. Patients with high risk organ involvement and skin, lymphadenopathy and pleural involvement respectively obtained sustained partial remissions. At median 33 months follow-up one patient had died from a stroke unrelated to $\mathrm{LCH}$.

Schini et al. [28] described five adult patients with relapsed or refractory $\mathrm{LCH}$, who received cladribine at a dose of $0.14 \mathrm{mg} /$ $\mathrm{kg} / \mathrm{d}$ as 2-hour infusions for 5 days every 4 weeks. One patient with lung involvement and repetitive pneumothorax was free of pneumothorax at 20 months follow-up. One patient with multisystem involvement obtained substantial improvement of disease. One patient with multiple bone lesions obtained impressive improvement, still present at 33 months followup. One patient with bone, lung and cerebellar involvement obtained significant improvement with disappearance of the cerebellar lesion. One patient with multiple bone lesions achieved stabilization of the disease, still sustained at 5 years follow-up.

Saven et al. [29] reported three adult patients, two with relapsed cutaneous disease and one with skin lesions, lymphadenopathy and hepatosplenomegaly. The patients received cladribine 0.7 $\mathrm{mg} / \mathrm{kg}$ per course given intravenously over 5 to 7 days. Patients with cutaneous lesions had been pretreated with vinblastine without and with other chemotherapy and received two and five courses respectively, and the patient with multisystem disease, who was treatment naïve, with two courses. All three patients obtained complete remissions and were disease free at respectively 5 months, 23 months and 15 months follow-up.

Vanicek et al. [30] reported two adults who had diabetes insipidus due to pituary stalk infiltration by LCH. Cladribine $5 \mathrm{mg} /$ $\mathrm{m}^{2} / \mathrm{d}$ subcutaneously for 5 days was administered for four and six cycles respectively; in the latter case during the last two cycles 20 $\mathrm{mg}$ dexamethasone was added. Both patients obtained complete remission and were disease free at 33 and 8 months follow-up. However both continued to require vasopressine and the first patients also testosterone.

Lajolo et al. [31] reported one adult with palatal and vulvar lesions, diagnosed as $\mathrm{LCH}$. The patient was treated with etoposide and radiotherapy and obtained partial remission with a duration of five months and relapsed with a lesion in the parotis. Cladribine $0.14 \mathrm{mg} / \mathrm{kg} / \mathrm{d}$ for 5 days was administered. After four cycles, the last two at reduced dose, complete remission was achieved. At the time of reporting, the patient remained in complete remission.

Dimopoulos et al. [32] reported a patient aged 34 years with $\mathrm{LCH}$ and multiple bone and intestinal lesions who was treated with four courses of cladribine at a dose of $0.1 \mathrm{mg} / \mathrm{kg} / \mathrm{d}$ for seven days by continuous intravenous infusion at 4 weeks interval. The patient obtained a complete response and was disease free at the 12 months follow-up evaluation.

Dallafior et al. [33] reported an adult patient with cutaneous $\mathrm{LCH}$, who had been treated with topical medications. Upon relapse the patient was treated with one cycle cladribine $0.1 \mathrm{mg} / \mathrm{kg} / \mathrm{d}$ intravenously for 7 days, which could not be continued due to severe neutropenia and anemia; a short complete response was achieved but the patient relapsed after one month. She was then treated with $200 \mathrm{mg}$ thalidomide and pentoxifylline and obtained a remission; upon relapse after 3 months, she continued on thalidomide and remained in remission at 6 months follow-up with $100 \mathrm{mg}$ thalidomide as maintenance therapy.

Robak et al. [34] reported and adult patient who had systemic lupus erythomatodes, and was diagnosed with LCH of the liver, spleen and a retroperitoneal lesion between the stomach and spleen. After failure of response to vincristine and prednisone cladribine $0.12 \mathrm{mg} / \mathrm{kg} / \mathrm{d}$ as 2-hour infusion for 3 days and cyclophosphamide $650 \mathrm{mg} / \mathrm{m} 2$ intravenously on day 1 was initiated. Five courses were administered with complete resolution of the lesions after the third course. At 6 months follow-up the patient was still in complete remission.

Buchler et al. [35] report an adult of twenty five years who had been diagnosed with diabetes insipidus based on LCH at five years of age. At the age of twenty five ataxia, dysarthria and slow speech developed. PET-FDG showed asymmetry of the brain stem. Cladribine $5 \mathrm{mg} / \mathrm{m} 2$ intravenously for five days was administered. Six cycles were given. After treatment the brain stem asymmetry disappeared, progression of neurological symptoms was arrested, but there was no regression of posterior fossa/cerebellar symptomatology.

Black et al. [36] reported an adult patient with pruritic rash, erythematous papules and ulcerated plagues. Cutaneous LCH was diagnosed. Cladribine intravenously for 7 days was administered, upon which the patient obtained complete response. At the time of reporting at least 2 months later, the patient was still in complete remission.

Gabalec et al. [37] reported an adult patient with panhypopituarism, suprasellar expansion, skeletal and retrobulbar infiltration, cervical, retroperitoneal and mediastinal lymphadenopathy, diagnosed as LCH. Five cycles of cladribine $0.14 \mathrm{mg} / \mathrm{kg} / \mathrm{d}$ for five days were administered. Complete remission of the lymphadenopathy and tumor infiltration was obtained but a lesion in the jejunum subsequently developed that induced bowel dilatation. After end-to-end anastomosis allogeneic stem cell transplantation was performed. At 130 days follow-up the patient was in good condition without any signs and symptoms of $\mathrm{LCH}$.

Table 4: Studies and case reports which included children and adults with LCH who were treated with cladribine.

Adam et al. [38] reported a survey conducted among patients with LHC who received treatment with cladribine. Of 60 adults $51(85 \%)$ achieved a complete or partial response and of 182 children $110(60.4 \%)$ obtained a complete or partial remission. The higher response in adults was among others attributed to the fact that $\mathrm{LCH}$ is less aggressive in adults than in children, but also the source of information was considered a factor. It was noted that cladribine is also effective in LCH patients with cranial involvement. Other authors reported good cranial response as well $[14,15,27,28,30]$. 
Table 4: Studies and case reports which included children and adults with LCH who were treated with cladribine.

\begin{tabular}{|c|c|c|c|c|c|c|c|}
\hline Ref) Author & Pretreatment & Treatment & $\begin{array}{c}\text { No of patients, } \\
\text { age }\end{array}$ & ORR & DFS & os & Type of LCH \\
\hline 38) Adam Z & & Cladribine & $\begin{array}{l}60 \text { adults } \\
182 \text { children }\end{array}$ & $\begin{array}{c}\text { ORR } 51(85 \%) \\
\text { ORR } 110(60.4 \%)\end{array}$ & & & Survey \\
\hline 39) Dhall G & $\begin{array}{l}2 \text { untreated } \\
10 \text { pretreated }\end{array}$ & $\begin{array}{l}\text { Cladribine i.v. } \\
\text { 5-13 mg/m2/d 5- } \\
\text { 3d, } 6 \text { cycles }\end{array}$ & $\begin{array}{c}8 \text { Kids } \\
4 \text { adults }\end{array}$ & $\begin{array}{l}\text { CR } 8 \text { (66\%) } \\
\text { ORR 100\% }\end{array}$ & $\begin{array}{l}\text { At } 2 \text { to } 10 \text { y FU } \\
\text { radiologic } 100 \%\end{array}$ & $\begin{array}{c}\text { At } 2 \text { to } 10 y \\
91.7 \%\end{array}$ & CNS LCH \\
\hline 40) Grau J & $\begin{array}{l}7 \text { untreated } \\
2 \text { pretreated }\end{array}$ & $\begin{array}{l}\text { Cladribine i.v. } \\
0.1 \mathrm{mg} / \mathrm{kg} / \mathrm{d} 5 \mathrm{~d} \text { x } \\
\text { cycles }\end{array}$ & $\begin{array}{c}9 \text { patients, med } \\
25(6-63) y\end{array}$ & $\begin{array}{l}2 \text { CR }(22 \%) \\
\text { ORR (66\%) }\end{array}$ & At report DFS 58\% & $\begin{array}{c}\text { At report OS } \\
71 \%\end{array}$ & Multiorgan LCH \\
\hline
\end{tabular}

ORR=overall response rate, $D F S=$ disease free survival, $O S=$ overall survival, $C R=$ complete respose, i.v.=intravenous, $y=y e a r, C N S=c e n t r a l$ nervous system

Dhall et al. [39] reported twelve patients, eight children from almost 3 to 13 years and four adults with $\mathrm{LCH}$ involving the hypothalamic-pituitary axis, parenchymal and dural lesions and choroid plexus based mass lesions. Ten of twelve patients with CNS involvement had multisystem disease. Nine patients had neurologic symptoms. Cladribine 5 to $13 \mathrm{mg} / \mathrm{m}^{2} / \mathrm{d}$ intravenously for 3 to 5 days was administered every 2 to 8 weeks for a period of 3 to 12 months. Complete radiologic response was obtained in eight (67\%) patients and partial radiologic response in four (33\%) patients. Response was achieved mean 9.4 months after initiation of cladribine. Two patients with partial response remained without progression for 10 years, whereas two patients progressed at 2 and 3 years respectively. One patient died from a status epilepticus, without evidence of $\mathrm{LCH}$ at autopsy. Permanent sequela of central nervous system $\mathrm{LCH}$, such as panhypopituitarism, diabetes insipidus and neurocognitive dysfunction were not reversible by cladribine therapy.

Grau et al. [40] reported nine patients, aged 6 to 63 years, who were diagnosed with multisystem $\mathrm{LCH}$ with severe organ dysfunction in four patients. Cladribine $0.1 \mathrm{mg} / \mathrm{kg} / \mathrm{d}$ for 5 days at 4 weeks interval was administered to seven treatment naïve and two patients in third line. Complete remission occurred in two (22\%) patients and partial remission in four (44\%) patients. The main toxicity was hematologic. At median 8 (range 2-17) months follow-up, two patients remained in complete and four patients in partial remission, while one had active disease. Two patients died due to progressive disease and yeast sepsis respectively. Probabilities of disease free and overall survival were $58 \%$ and $71 \%$ respectively.

Table 5: Studies and case reports of patients with pulmonary LCH who were treated with cladribine.

Grobost et al. [41] reported five adult patients with pulmonary $\mathrm{LCH}$. Two patients had received prednisolone and the other three patients were treatment naïve. Cladribine was administered at a dose of $0.1 \mathrm{mg} / \mathrm{kg} / \mathrm{d}$ subcutaneously for 5 days for three or four courses. Dyspnea improved after treatment in four patients and forced expiration volume in 1 second (FEV1) increased in all patients. Forced vital capacity recuperated with therapy by a mean of $414 \mathrm{ml}$ and FEV1 by a mean of $387 \mathrm{ml}$. Chest high resolution $\mathrm{CT}$ scans improved with cladribine treatment in four cases. Patients were followed for a median of 22 months after cladribine treatment. One patient relapsed after 6 months and was retreated with cladribine. Three other patients had a remission, one at evaluation four years after cladribine treatment, and one patient's function tests improved and the disease had stabilized two years after cladribine treatment.

Lorillon et al. [42] reported three adults with progressive cystic pulmonary $\mathrm{LCH}$. The patients had been pretreated with vinblastine and/or steroids but progressed. The patients received four or five cycles of cladribine $0.1 \mathrm{mg} / \mathrm{kg} / \mathrm{d}$ subcutaneously for 5 days. One patient of 21 years had diabetes insipidus and a biopsy proven osteolytic lesion of the skull and a decreased lung function. The dyspnea improved after treatment with cladribine and there was partial resolution of nodular and cystic lung lesions. One patient of 32 years with only lung lesions obtained resolution of abnormalities and improvement of DLCO value by $46 \%$ compared to pretreatment. One patient of 23 years had multisystem $\mathrm{LCH}$ since childhood; the bullocystic lung lesions almost completely resolved after treatment with cladribine and the lung function improved.

Goh et al. [43] reported a 19 year old patient with pulmonary LCH who received four cycles of cyclophosphamide, doxorubicine, vincristrine and prednisolone (CHOP) with etoposide which induced no improvement of symptoms. Subsequently he received five cycles of cladribine $0.1 \mathrm{mg} / \mathrm{kg} / \mathrm{d}$ by continuous infusion for 7 days per course over a period of ten months. The patient experienced rapid improvement of performance and more gradual improvement of the lung function. Five years after the last treatment he remained well free of symptoms.

Aerni et al. [44] reported a sixty six year old patient, who presented with cervical lymphadenopathy, discrete pulmonary nodes and cervical and thoracic vertebral body lesions, diagnosed as $\mathrm{LCH}$. Cladribine $5 \mathrm{mg} / \mathrm{m} 2 / \mathrm{d}$ intravenously for 5 days every 4 weeks for four cycles was administered. A complete response occurred and at 18 months follow-up there was no evidence of LCH in lungs, spine and lymph nodes.

Lazor et al. [45] reported one adult presenting with dry cough, diagnosed with $\mathrm{LCH}$. The lungs contained nodular cystic lesions. During prednisone treatment the lung function deteriorated. It was decided to instate cladribine. She received four cycles of 3 , 4,5 and $5 \mathrm{mg} / \mathrm{m} 2$ every 4 weeks. The CT and dyspnea improved, while she remained smoking. 
Table 5: Studies and case reports of patients with pulmonary LCH who were treated with cladribine.

\begin{tabular}{|c|c|c|c|c|c|c|c|}
\hline Ref) Author & Pretreatment & Treatment & $\begin{array}{c}\text { No of patients, } \\
\text { age }\end{array}$ & ORR & DFS & os & Type of LCH \\
\hline 41) Grobost V & $\begin{array}{c}\text { Prednisolone in } 2 \\
\text { patients }\end{array}$ & $\begin{array}{c}\text { Cladribine s.c. } \\
0.1 \mathrm{mg} / \mathrm{kg} / \mathrm{d} \text { 5d 3-4 cycles }\end{array}$ & $\begin{array}{l}5 \text { adults, aged } \\
\text { median } 42.5 \mathrm{y}\end{array}$ & ORR $100 \%$ & $\begin{array}{c}\text { At } 1 \text { y } 4(80 \%) \\
\text { DFS }\end{array}$ & & Pulmonary LCH \\
\hline 42) Lorillon G & $\begin{array}{l}\text { Vinblastine } \\
\text { Steroids }\end{array}$ & $\begin{array}{l}\text { Cladribine s.c. } \\
0.1 \mathrm{mg} / \mathrm{kg} / \mathrm{d} 5 \mathrm{~d} \times \text { cycles }\end{array}$ & $\begin{array}{l}3 \text { adults, aged } \\
21,23,32 \text { y }\end{array}$ & ORR $100 \%$ & & & Pulmonary LCH \\
\hline 43) Goh NS & $\begin{array}{l}4 \text { cycles } \mathrm{CHOP} \\
\text { etoposide }\end{array}$ & $\begin{array}{l}\text { Cladribine i.v. } \\
0.1 \mathrm{mg} / \mathrm{kg} / \mathrm{d} 7 \mathrm{~d} \\
5 \text { cycles }\end{array}$ & 1 pat, aged $19 \mathrm{y}$ & $1 \mathrm{CR}$ & At $5+y$ DFS & & $\begin{array}{l}\text { Pulmonary and } \\
\text { skeletal LCH }\end{array}$ \\
\hline 44) Aerni MR & untreated & $\begin{array}{c}\text { Cladribine i.v. } 5 \mathrm{mg} / \mathrm{m} 2 / \mathrm{d} 5 \mathrm{~d} \\
4 \text { cycles }\end{array}$ & $\begin{array}{c}1 \text { adult, aged } \\
66 y\end{array}$ & $1 \mathrm{CR}$ & At $18 \mathrm{~m}$ DFS & & $\begin{array}{l}\text { Pulmonary and } \\
\text { systemic LCH }\end{array}$ \\
\hline 45) Lazor R & Steroids & $\begin{array}{c}\text { Cladribine } 3-5 \mathrm{mg} / \mathrm{m} 2 \\
4 \text { cycles }\end{array}$ & $\begin{array}{c}1 \text { adult, aged } \\
39 y\end{array}$ & 1 ORR & & & Pulmonary LCH \\
\hline 46) Epaud R & $\begin{array}{l}\text { Vinblastine } \\
\text { Steroids } \\
\text { 6-MP }\end{array}$ & $\begin{array}{l}\text { Cladribine s.c. } 5 \mathrm{mg} / \mathrm{m} 2 / \mathrm{d} 5 \mathrm{~d} \\
6 \text { cycles }\end{array}$ & $\begin{array}{c}1 \text { child, aged } \\
13 y\end{array}$ & $1 \mathrm{ORR}$ & & & $\begin{array}{l}\text { Pulmonary and } \\
\text { systemic LCH }\end{array}$ \\
\hline 47) Lorillon G & Vinblastine & Cladribine s.c. & $\begin{array}{c}1 \text { adult, aged } \\
23 y\end{array}$ & 1 near CR & $\begin{array}{c}\text { At } 4 \text { years FU } \\
\text { DFS }\end{array}$ & & $\begin{array}{l}\text { Pulmonary and } \\
\text { systemic LCH }\end{array}$ \\
\hline 48) Nassar M & untreated & $\begin{array}{c}\text { Cladribine s.c. } \\
0.1 \mathrm{mg} / \mathrm{kg} / \mathrm{d} 5 \mathrm{~d} 4 \text { cycles }\end{array}$ & $\begin{array}{c}1 \text { adult, aged } \\
34 \mathrm{y}\end{array}$ & $1 \mathrm{CR}$ & & & $\begin{array}{l}\text { Pulmonary and } \\
\text { systemic LCH }\end{array}$ \\
\hline
\end{tabular}

$\mathrm{ORR}=$ overall response rate, $\mathrm{DFS}=$ disease free survival, $\mathrm{OS}=$ overall survival, $\mathrm{CR}=$ complete response, $\mathrm{CHOP}=\mathrm{cyclophosphamide,} \mathrm{adriamycine,} \mathrm{vincristine,}$ prednisone, 6-MP=6-mercaptopurine, i.v.=intravenous, s.c.=subcutaneous, $y=y e a r, F U=$ follow-up

Epaud et al. [46] reported a child with cystic pulmonary LCH. $\mathrm{LCH}$ had been diagnosed by liver and skin biopsy; the organ involvement resolved upon treatment with vinblastine, steroids and 6- mercaptopurine for 15 months. Two months after the end of treatment reactivation of disease was diagnosed with mediastinal node and lung involvement. Lung involvement was limited to a few cysts and vinblastine and steroid treatment were resumed. The lung cysts resolved, but 2 months later the patient presented with diabetes insipidus and thyroid insufficiency. Hormone replacement therapy was given. Eighteen months later, reactivation of the lung disease occurred with bilateral pneumothorax. Surgical pleurodesy and treatment with vinblastine and 6-mercaptopurine was given for 12 months, with no change in CT finding or clinical stability. At 13 years of age, reactivation of the lung disease occurred, and the patient presented with dyspnea and deterioration of the lung function. Cladribine $5 \mathrm{mg} / \mathrm{m} 2 / \mathrm{d}$ subcutaneously for five days every 4 weeks was administered during six cycles. At 2.5 years after start of cladribine therapy the dyspnea had improved, the cystic lesions had decreased or resolved and the lung function parameters improved by more than $50 \%$.

Lorillon et al. [47] reported one adult patients with multisystem $\mathrm{LCH}$. At four years of age the patient had presented with skin, lymph node and pituitary stalk involvement with diabetes insipidus. Vinblastine induced complete remission. At 22 years of age cutaneous and lymph node LCH lesions recurred, and lung involvement was diagnosed. As vinblastine was not effective, treatment with cladribine subcutaneously was started. Skin and lymph node lesions resolved and impressive lung improvement occurred. At follow-up 4 years later he was asymptomatic and only received desmopressin for diabetes insipidus.

Nassar et al. [48] reported one adult patient with diabetes insipidus based on a hypophyseal microadenoma and advanced cystic pulmonary LCH. Despite cessation of smoking the lung function deteriorated and subcutaneous cladribine $0.1 \mathrm{mg} / \mathrm{kg} / \mathrm{d}$ for 5 days during four cycles was administered. After treatment there was dramatic improvement of the lung function tests and radiologic abnormalities. At one year the patient remained in remission.

\section{Discussion}

Our summary presents cladribine use in children and adults with cutaneous, focal, multisystem, pulmonary and in low, intermediate and high risk $\mathrm{LCH}$. Children were treated with cladribine monotherapy (Table 1 ) or cladribine and cytarabine (Table 2). In children cytarabine is often added when the disease is multisystem and high risk organs are involved (16-22), but also cladribine monotherapy has been used for high risk organ involvement $[6,8,10,11]$. In general cladribine plus or minus cytarabine is administered in second line after prior treatment with vinblastine and steroids, with or without other drugs [614,16-20,22]. There are two reports in which cladribine was administered in first line: a case of a brain stem tumor in which cladribine induced a sustained partial response and a case of multisystem $\mathrm{LCH}$, in which cladribine was given in combination with vinblastine and prednisone; this combination induced a partial response, that was turned in a complete response when cladribine was combined with cytarabine [15,21]. Cladribine monotherapy induced in many cases complete durable response [6-8,10-14]. High response rates in children were also observed with the combination of cladribine and cytarabine. It is notable that this combination of drugs is only used in children and not in adults (Tables 1-5). This is due to the fact that childhood $\mathrm{LCH}$ often has a more aggressive course than adult $\mathrm{LCH}$. 
In adults cladribine monotherapy is more often used in first line than in children $[23-27,29,30,36,37]$.

Cladribine penetrates the blood brain barrier. Cladribine is therefore used in first line when there is central nervous system involvement and induces promising responses [27,30,35,37]. In adults high durable complete response rates are obtained with cladribine monotherapy [25-30,32,34]. Although cladribine is seldomly used when there is cutaneous involvement only, in one case a complete remission was reported after one course of treatment [36]. Cutaneous lesions respond well to cladribine $[24,36]$. LCH of the pituitary stalk was often reported $[13,30,37,39]$. Although mass lesions generally resolve with cladribine, resolution of hormonal deficiency rarely occurs and requires lifelong hormone replacement therapy. Table 4 shows publications in which children and adults are jointly included [3840]. The results show that cladribine is efficient in children as well as in adults. The survey by Adams et al. suggest a higher overall response rate in adults then in children, but this may depend on the type of disease, low, intermediate or high risk, and single or multisystem involvement more than the age. Pulmonary LCH is a distinct entity that often occurs in smokers [41-48]. Although smoking cessation alone may lead to regression, cladribine is often used either in first or second line and has been reported to induce dramatic improvement of nodular and cystic lesion and improvement of lung function also at long term follow-up [41-48]. Cladribine has generally been used intravenously in $\mathrm{LCH}$ however subcutaneous use has been reported $[12,25,26,30]$. Cladribine administered subcutaneously is as efficient as intravenous administration $[49,50]$. In the publications in which subcutaneous use was reported, almost all patients obtained complete response $[12,25,26,30]$. The doses of cladribine varied considerable. In children with multiorgan involvement in whom cladribine was used in combination with cytarabine the dose exceeded generally $5 \mathrm{mg} / \mathrm{m}^{2} / \mathrm{d}$ for 5 days per cycle (Table 2), whereas the dose of $5 \mathrm{mg} / \mathrm{m}^{2} / \mathrm{d}$ or 0.1 to $0.14 \mathrm{mg} / \mathrm{kg} / \mathrm{d}$ for 7 to 5 days per cycle is a dose commonly used as monotherapy in children and adults regardless of the extend of the disease (Tables 1, 3-5).

\section{Conclusion}

Cladribine is highly efficient in first line and second line treatment for relapsed or refractory $\mathrm{LCH}$, either alone or in combination with cytarabine and can be administered subcutaneously or intravenously.

\section{References}

1. Carstensen H, Ornvold K (1993) The epidemiology of Langerhans cell histiocytosis in children in Denmark 1975-1989. Med Pediatr Oncol 21: 387.

2. McClain ML, Allen CE (2010) Inflammatory and malignant histiocytosis. In Williams Hematology 2010, 8th ed. pubs Mc Graw Hill Medical, p: 1049.

3. Haupt R, Minkov M, Astigarraga I, Schafer E, Nanduri V, et al. (2013) Langerhans cell histiocytosis: Guidelines for diagnosis, clinical workup and treatment for patients till the age of 18 years. Pediatr Blood Cancer 60: 175-184.
4. Girschikofsky M, Arico M, Castillo D, Chu A, Doberauer C, et al. (2013) Management of adult patients with Langerhans cell histiocytosis: recommendation from an expert panel on behalf of Euro-Histio-Net. Orphanet J rare Dis 8: 72.

5. Digal DS, Miller HJ, Schram ED, Saven A (2010) Beyond hairy cell: the activity of cladribine in other hematologic malignancies. Blood 116 2884-2896.

6. Donadieu J, Bernard F, van Noesel M, Barkaoui M, Bardet O, et al. (2015) Cladribine and cytarabine in refractory multisystem Langerhans cell histiocytosis: results of an international phase 2 study. Blood 126: 1415-1423.

7. Mottl H, Stary J, Chanova M, Nekola M, Drahokoupilova E, et al. (2006) Treatment of recurrent Langerhans cell histiocytosis in children with 2-chlorodeoxyadenosine. Leuk Lymphoma 47: 1881-1884.

8. Stine KC, Saylors RL, Saccente S, McClain KL, Becton DL, et al. (2004) Efficacy of continuous infusion 2-CdA (cladribine) in pediatric patients with Langerhans cell histiocytosis. Pediatr Blood Cancer 43: 81-84.

9. Biswas G, Khadwal A, Arora B, Bhagwat R, Banavali SD, et al. (2007) Activity and toxicity of 2-CDA in Langerhans cell histiocytorsis: A single institutional experience. Indian J Cancer 44: 137-141.

10. Rodigruez-Galindo $C$, Kelly $P$, Jeng M, Presbury GG, Rieman $M$, et al. (2002) Treatment of children with Langerhans cell histiocytosis with 2-chlorodeoxyadenosine. Am J Hematol 69: 179-184.

11. Stine KC, Saylors RL, Williams LL, Becton DL (1997) 2-Chlorodeoxyadenosine (2-CDA) for the treatment of refractory or recurrent Langerhans cell histiocytosis in pediatric patients. Med \& Pediatr Oncol 29: 288-292.

12. Baumann M, Cerny T, Sommacal A, Koeberle D (2011) Langerhans cell histiocytosis with central nervous system involvement-complete response to 2-chlorodeoxy adenosine after failure of tyrosine kinase inhibitor therapies with sorafenib and imatinib. Hematol Oncol 30: 101-104.

13. Ottaviano F, Finlay JL (2003) Diabetes Insipidus and Langerhand cell histiocytosis: a case report of reversibility with 2-chlorodeoxyadenosine. J Pediat Hematol Oncol 25: 575-577.

14. Suzuki D, Kobayashi R, Sano H, Kishimoto K, Yasuda K, et al. (2013) Spontaneous remission of 2-chlorodeoxyadenosine-related secondary myelodysplastic syndrome in a patient with refractory Langerhand cell histiocytosis. In J hematol 97: 782-785.

15. Savardekar A, Tripathi M, Bansal D, Vaiphei K, Gupta SK, et al. (2013) Isolated tumorous Langerhand cell histiocytosis of the brainstem: a diagnostic and therapeutic challenge. J Neurosurg Pediatr 12: 258-261.

16. Rigaud C, Barkaoui MA, Thomas C, Bertrand Y, Lambilliotte A, et al. (2016) Langerhans cell histiocytosis: therapeutic strategy and outcome in a 30-year nationwide cohort of 1478 patients under 18 years of age. Br J Haematol 174: 887-898.

17. Hu T, Liu R, Li J, Cao J, Zhang L, et al. (2014) Cladribine for 13 cases refractory high-risk children Langerhans cell histiocytosis. Zhonghua Xue Ye Xue Za Zhi 35: 985-989.

18. Bernard F, Thomas C, Bertrand Y, Munzer M, Parker JL, et al. (2005) Multicenter pilot study of 2 chlorodeoxyadenosine and cytosine arabinoside combined chemotherapy in refractory langerhans cell histiocytosis with haematological dysfunction. Eur J cancer 41: 2682-2689.

19. Rosso DA, Amaral D, Latella A, Chantada G, Braier JL, et al. (2016) Reduced doses of cladribine and cytarabine regimen was effective and well tolerated in patient with refractory-risk multisystem Langerhand cell histiocytosis. Br J Haematol 172: 287-290. 
20. Apollonsky N, Lipton JM (2009) Treatment of refractory Langerhans cell histiocytosis with a combination of 2-chlorodeoxyadenosine and cytosine arabinoside. J Pediatr Hematol Oncol 31: 53-56.

21. Choi SW, Bangaru BS, Wu CD, Finlay JL (2003) Gastronintestinal involvement in disseminated Langerhans cell histiocytosis with durable complete response to 2-chlorodeoxyadenosine and highdose cytarabine. J Pediatr Hematol/Oncol 25: 503-506.

22. Yao X, Ying H, Weihong Z, Qing S, Xintian L, et al. (2015) Cladribine treatment of repeatedly relapsed Langerhas cell histiocytosis: a case report and review of the literature. Zhonghua Er Za Zhi 53: 296-299.

23. Cantu MA, Lupo PJ, Bilgi M, Hicks MJ, Allen CE, et al. (2012) Optimal therapy for adults with Langerhans cell histiocytosis bone lesions. PLoS ONE 7: e43257.

24. Saven A, Burian C (1999) Cladribine activity in adult Langerhans cell histiocytosis. Blood 93: 4125-4130.

25. Adam Z, Szturz P, Duras J, Pour L, Kreji M, et al. (2012) Treatment of Langerhans cell histiocytosis by cladribine reached long-term complete remission in 9 out of 10 adult patients. Klin Onkol 25: 255 261.

26. Adam Z, Szturtz P, Vanicek J, Moulis M, Pour L, et al. (2013) Cladribine (2- chlorodeoxyadenosine) in frontline chemotherapy for adult Langerhans cell histiocytosis: a single center study of seven cases. Acta Oncologica 52: 994-1001.

27. PardananiA, Phyliky RL, LICY, TefferiA(2003) 2-Chlorodeoxyadenosine therapy for disseminated Langerhans cell histiocytosis. Mayo Clin Proc 78: 301-306.

28. Schini M, Makras P, Kanakis G, Voulgarelis M, Kaltsas G, et al. (2013) Cladribine therapy in adult patients with advanced Langerhans cell histiocytosis. Leuk Lymphoma 54: 1541-1543.

29. Saven A, Foon KA, Piro LD (1994) 2-Chlorodeoxyadenosine-induced complete remissions in Langerhand cell histiocytosis. Ann Internal Med 121: 430-432.

30. Vanicek J, Adam Z, Balsicova K, Kreici M, Pour L, et al. (2011) MRdocumented remission of pituary stalk infiltration in patients with Langerhans cell histiocytosis following treatment with 2-chlorodeoxyadenosine. Vnit Lek 57: 671-675.

31. Lajolo C, Campisi G, Deli G, Littarru C, Guiglia R, et al. (2012) Langerhans's cell histiocytosis in old subjects: two rare case reports and a review of the literature. Gerodontology 29: e1207-e1214.

32. Dimopoulos MA, Theodorakis M, Kostis E, Papadimitris C, Moulopoulos LA, et al. (1997) Treatment of Langerhans cell histiocytosis with 2-chlorodeoxyadenosine. Leuk Lymphoma 25 187-189.

33. Dallafior S, Pugin P, Cerney T, Betticher D, Suarat JH, et al. (1995) Erfolgreiche behandlung eins Falls von kutaner Langerhans-zellgranulomatose mit 2-chlorodeoxyadnosine und thalidomid. Hautarzt 46: 553-560.

34. Robak T, Kordez R, Tobak E, Bartkowiak J, Biernat W, et al. (2002) Langerhans cell histiocytosis in a patient with systemic lupus erythomatosus : a clonal disease responding to treatment with cladribine and cyclophosphamide. Leuk Lymphoma 43: 2041-2046.

35. Buchler T, Cervinek L, Belohlavwk O, Kantorova I, Mechl M, et al. (2005) Langerhans cell histiocytosis with central nervous system involvment : follow-up by FDG-PET during treatment with cladribine. Pediatr Blood Cancer 44: 286-288.

36. Black A, Bershow A, Allen PS, Crowson AN (2011) Seventy nine year old man with Langerhans cell histiocytosis treated with cladribine. J Am Acad Dermatol 65: 681-683.

37. Gabalec F, Simkovic M, Zavrelova A, Kasparova P, Moravkova $P$, et al. (2017) Treatment of multifocal multisystem BRAF positive Langerhans cell histiocytosis with cladribine, surgery and allogeneic stem cell transplantation. Acta medica 60: 152-156.

38. Adam Z, Szturz P, Pour L, Kreji M, Zahradova L, et al. (2012) Cladribine is highly effective in the treatment of Langerhans cell histiocytosis and rare histiocytic disorders of the juvenile xanthogranuloma group. Vnitr Lek 58: 455-465.

39. Dhall G, Finlay JL, Dunkel IJ, Ettinger LJ, Kellie SJ, et al. (2008) Analysis of outcome for patients with mass lesions oft he cntral nervous system due to Langerhans cell histiocytosis treated with 2-chlorodeoxyadenosine. Pediatr Blood Cancer 50: 72-79.

40. Grau J, Ribera JM, Tormo M, Indiano JM, Vercher J, et al. (2001) Results of treatment with 2-chlorodeoxyadenosine in refractory or relapsed Langerhans cell histiocytosis. Study of 9 patients. Med Clin 116: 339-342.

41. Grobost V, Khouatra C, Lazor R, Cordier JF, Cottin V, et al. (2014) Effectiveness of cladribine therapy in patients with pulmonary Langerhans cell histiocytosis. Orphanet J Rare Dis 9: 191.

42. Lorillon G, Bergeron A, Detourmignies L, Jouneau S, Wallaert B, et al. (2012) Cladribine is effective against cystic pulmonary Langerhans cell histiocytosis. Am J Resp and Critical Care Med 186: 930-932.

43. Goh NS, McDonald CF, MacGreor DP, Pretto JJ, Brodie GN, et al. (2003) Successful treatment of Langerhans cell histiocytosis with 2-chlorodeoxyadenosine. Respiratory 8: 91-94.

44. Aerni MR, Aubry MC, Myers JL, Vassallo R (2008) Complete remission of nodular pulmonary Langerhans cell histiocytosis lesions induced by 2-chlorodeoxyadenosine in a non-smoker. Respir Med 102: 316319.

45. Lazor R, Etienne-Mastroianni B, Khouatra C, Tazi A, Cottin V, et al. (2009) Progressive diffuse pulmonary Langerhans cell histiocytosis improved by cladribine chemotherapy. Thorax 64: 274-275.

46. Epaud R, Ducou le Pointe H, Fasola S, Okiyssard S, Delestrain C, et al. (2015) Cladribine improves lung cysts and pulmonary function in a child with histiocytosis. Eur Repir J 45: 831-833.

47. Lorillon G, Tazi A (2017) How I manage pulmonary Langerhans cell histiocytosis. Eur Respir Rev 26: 170070.

48. Nassar M, Traclet J, Cottin V (2018) Effect of cladribine therapy on lung cysts in pulmonary Langerhans cell histiocytosis. ERJ Open Res 4: 00089-2017.

49. Von Rohr A, Schmitz SFH, Tichelli A, Hess U, Piguet D, et al. (2002) Treatment of hairy cell leukemia with cladribine by subcutaneous bolus injection: a phase II study. Ann Oncol 13: 1641-1649.

50. Khorsid O Namour AE, El-Gammal MM, Mahmoud TY, Fortpied C, Abdel-Malek R, et al. (2015) Efficacy and Safety of Cladribine: Subcutaneous versus intravenous administration in hairy cell leukemia patients. Mediterr J Hematol Infect Dis 7: e2015058. 